\section{Presumed Delayed Onset Trochle- ar Nerve Palsy after Endovascular Treatment for the Aneurysm in Cavernous Portion of Internal Ca- rotid Artery}

Dear Editor,

The endovascular technique is a minimally invasive, effective method for the evaluation and treatment of aneurysm in the cavernous portion of the internal carotid artery (ICA) [1,2]. Delayed-onset cranial nerve palsy after endovascular treatment has rarely been reported [3]. We report a case of delayed-onset trochlear nerve palsy after endovascular treatment of an aneurysm in the cavernous portion of the ICA.

A 77-year-old woman visited our clinic with a complaint of sudden-onset vertical diplopia 2 months prior. She reported experiencing gradual worsening of diplopia. The symptoms did not show diurnal variation or any relationship with fatigue. A large unruptured aneurysm in the cavernous portion of the left ICA was discovered incidentally on magnetic resonance imaging (MRI) while investigating headaches 7 years prior (Fig. 1A). The patient underwent endovascular coil embolization without any complications. She did not have any neurologic complications prior to the development of diplopia. She had hypertension and no other relevant medical history. There were no ocular injections, chemosis, pain, proptosis, abnormal lid problems, or visual disturbances in either eye. The patient's visual acuity was 20 / 25 in both eyes. The pupils showed a normal response to both light and near stimulation. Extraocular examination revealed 6 prism diopters left hypertropia in primary gaze with elevation during adduction of the left eye. The Bielschowsky head-tilt test revealed 8 prism diopters left hypertropia on left tilt and orthotropia on right tilt. Fundus examination revealed

Received: August 2, 2019 Final revision: November 13, 2019 Accepted: December 9, 2019 excyclotorsion of the left eye (Fig. 1B). There was no definite recanalization of the coiled aneurysm on three-dimensional time-of-flight MRI (Fig. 1C). Serologic tests including a thyroid function test and anti-acetylcholine receptor antibody test were conducted to evaluate the cause of diplopia, but the results were normal. Prism glasses were prescribed to alleviate diplopia. At the 10-month follow-up, the patient's symptoms improved with prism glasses, but the ocular motility findings remained unchanged (Fig. 1D).

The cavernous sinus contains the oculomotor nerve, trochlear nerve, ophthalmic and maxillary branches of the trigeminal nerve, abducens nerve, and ICA [4]. In the cavernous sinus, the ocular motor cranial nerves and vascular structures are located in close proximity to each other [4]. The "mass effect" of the enlarging aneurysm on the local neural structures or disruption of the blood supply to these structures may result in diplopia and pain [1].

In this case, trochlear nerve palsy developed 7 years after the procedure, without definitive recanalization on neuroimaging. Xu et al. [3] reported three patients with delayed-onset cranial nerve palsy after coiling of an aneurysm in the cavernous portion of the ICA. Their patients developed partial oculomotor nerve palsy with or without abducens nerve palsy at least 1 year after the procedure; recurrent filling or recanalization of the aneurysm was confirmed on subsequent angiography. Follow-up MRI in our case showed no residual filling within the aneurysm; unfortunately, subsequent angiography was not performed.

The possibility of microvascular ischemia should be considered in elderly patients. Trochlear nerve palsy from ischemia may improve over several weeks, or may not $[4,5]$. Our patient did not show any improvement over a follow-up period of nearly 1 year. An earlier study suggested that persistent mass or dynamic effects, exacerbated by remnant or recurrent flow across the neck of a coiled aneurysm, are the cause of delayed-onset cranial nerve palsy [3]. We postulated that the minimal dynamic changes in the coil-packed aneurysm, located anterosuperiorly within the cavernous sinus, may affect ocular motor cranial nerves or their vascular components. Since the trochlear nerve runs forward within the lateral wall of the cavernous sinus, anteriorly it crosses over the oculomotor nerve to enter the 

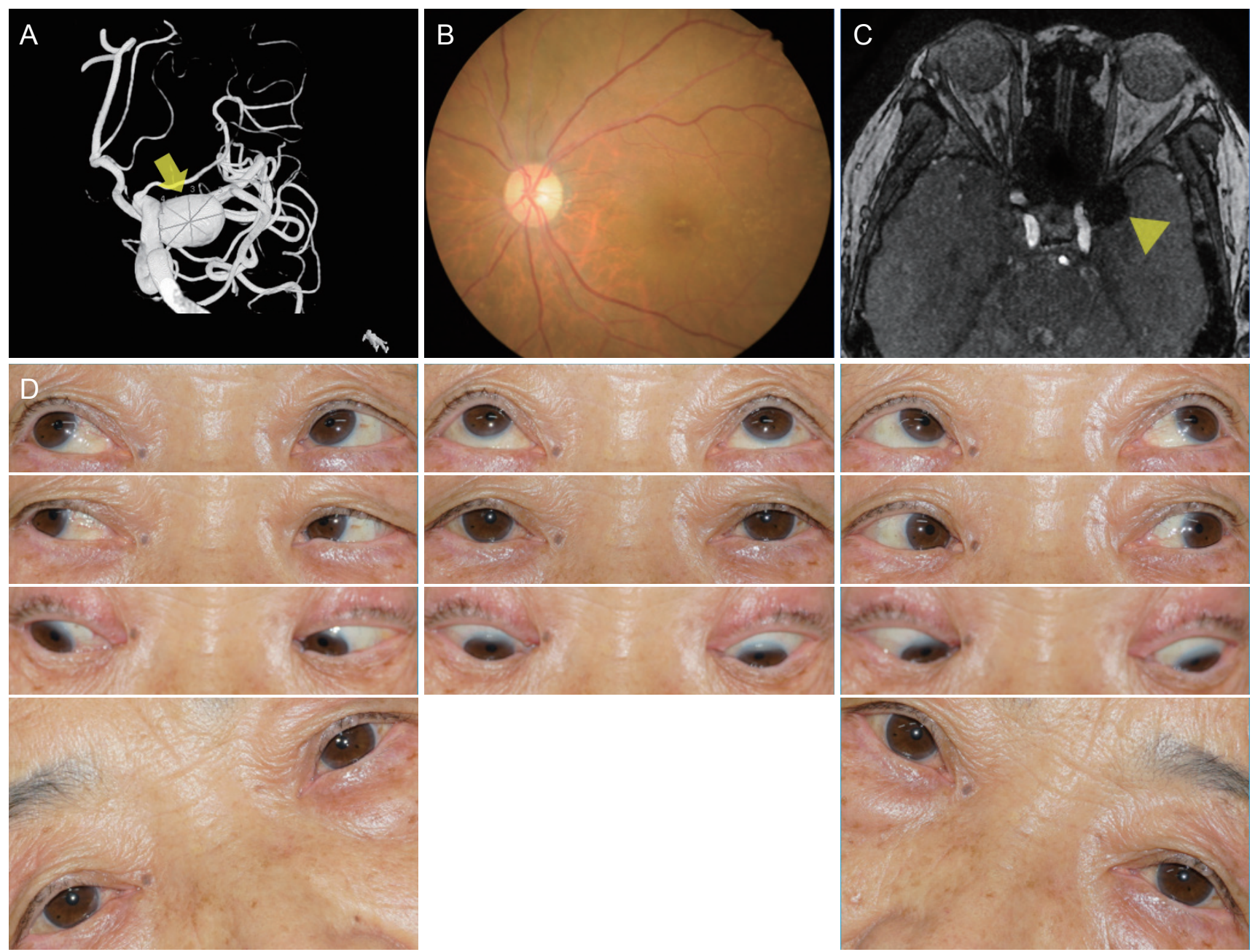

Fig. 1. Magnetic resonance imaging (MRI), fundus photo, and diagnostic gaze photos of patient. (A) MRI showing a large unruptured aneurysm in the cavernous portion of the left internal carotid artery (arrow, about $15 \mathrm{~mm}$ in diameter. (B) Fundus examination showing excyclotorsion of the left eye. (C) Axial view of three-dimensional time-of-flight MRI performed for investigation of diplopia revealed no residual filling or coil compaction within the aneurysm (arrowhead). (D) Image of the nine diagnostic gaze positions and tilting. The patient exhibited left hypertropia in the primary position with elevation during adduction of the left eye. Left hypertropia increased in the left tilt position. Patient provided written consent for the use of images.

superior orbital fissure [4]. The anatomical characteristics of the aneurysm in this case might have caused trochlear nerve palsy.

Jong Hoon Kim

Department of Neurosurgery, Yeungnam University College of Medicine, Daegu, Korea

\section{Won Jae Kim}

Department of Ophthalmology, Yeungnam University College of Medicine, Daegu, Korea

E-mail:eyekwj@ynu.ac.kr

\section{Conflict of Interest}

No potential conflict of interest relevant to this article was reported.

\section{References}

1. Starke RM, Chalouhi N, Ali MS, et al. Endovascular treatment of carotid cavernous aneurysms: complications, outcomes and comparison of interventional strategies. $J$ Clin Neurosci 2014;21:40-6. 
2. Stiebel-Kalish H, Kalish Y, Bar-On RH, et al. Presentation, natural history, and management of carotid cavernous aneurysms. Neurosurgery 2005;57:850-7.

3. Xu DS, Hurley MC, Batjer HH, Bendok BR. Delayed cranial nerve palsy after coiling of carotid cavernous sinus aneurysms: case report. Neurosurgery 2010;66:E1215-6.
4. Miller NR, Subramanian PS, Patel VR, editors. Walsh and Hoyt's clinical neuro-ophthalmology: the essentials. 3rd ed. Philadelphia: Wolters Kluwer; 2016. p. 341-71.

5. Galtrey CM, Schon F, Nitkunan A. Microvascular non-arteritic ocular motor nerve palsies: what we know and how should we treat? Neuroophthalmology 2014;39:1-11. 